- The general practitioner has an important role in the management of hypodontia.

- The severity of hypodontia does not indicate the complexity of treatment required.

- The general practitioner role is vital for maintenance following comprehensive treatment.

- Good liaison between general practitioner and dental professionals managing the case is important.

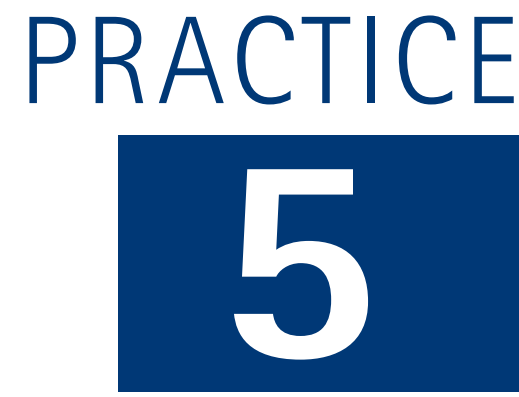

\title{
The interdisciplinary management of hypodontia: the relationship between an interdisciplinary team and the general dental practitioner
}

\author{
R. S. Hobson, ${ }^{1}$ N. E. Carter, ${ }_{1}^{2}$ T. J. Gillgrass, ${ }^{3}$ N. J. A. Jepson, ${ }^{4}$ J. G. Meechan, ${ }^{5}$ F. Nohl, ${ }^{6}$ and J. H. Nunn, ${ }^{7}$
}

This paper will illustrate how the general dental practitioner can provide care for patients with hypodontia. It will demonstrate how an interdisciplinary team works for hypodontia patients and, in particular, for those with severe hypodontia.

THE INTERDISCIPLINARY

MANAGEMENT OF

HYPODONTIA:

1. Paediatric dentistry

2. Restorative dentistry

3. Orthodontics

4. Oral surgery

5. The relationship between an interdisciplinary team and the general dental practitioner

${ }^{1}$ Senior Lecturer/Honorary Consultant in Orthodontics, ${ }^{2}$ Consultant in

Orthodontics, ${ }^{4}$ Senior Lecturer/Honorary Consultant in Restorative Dentistry,

${ }^{5}$ Senior Lecturer/Honorary Consultant in Oral Surgery, School of Dental Sciences, Framlington Place, Newcastle upon Tyne NE2 4BW, England: ${ }^{6}$ Consultant in

Restorative Dentistry, Newcastle Dental Hospital, Richardson Road, Newcastle upon Tyne NE2 4AZ: ${ }^{3}$ Consultant in Orthodontics, Edinburgh Postgraduate Institute, Lauriston Building, Lauriston Place, Edinburgh EH3 9YW, Scotland; ${ }^{7}$ Professor and Head of Department. Department of Public and Child Denta Health, Dental School and Hospital, Lincoln Place, Dublin 2, Ireland

Correspondence to: R. S. Hobson, Child Dental Health, Newcastle Dental School Framlington Place, Newcastle upon Tyne, England NE2 4BW

E-mail:R.S.Hobson@ncl.ac.uk

Refereed Paper

๑ British Dental Journal 2003; 194

479-482
The importance of a team approach to the management of complex hypodontia cases cannot be overemphasized. It is essential to provide an overall treatment plan whose aims are understood by each team member to ensure appropriate timing and management of each stage of treatment. This can only be achieved by close liaison and often involves simultaneous participation in treatment.

However, despite the need for close working within a team, the general dental practitioner with the appropriate skills can provide or contribute to high quality care for hypodontia patients and fulfill a number of key roles in patient care:

\section{IDENTIFICATION AND REFERRAL}

The general dental practitioner is usually the first to identify the absence of teeth as part of ongoing patient care. The practitioner may be aware of family circumstances which could affect the incidence of hypodontia; parents or siblings who have absent teeth and an awareness of hereditary conditions that may predispose to hypodontia. ${ }^{1}$ Ectopic tooth position and abnormal tooth shape are often associated with absent teeth, and these findings should prompt further examination. It is known that hypodontia has a genetic basis and other family members are often affected. Screening affected families is important to ensure identification and appropriate timing of interventions.

There are a number of key ages at which hypodontia patients may present:

\section{Before 6 years}

In this age group primary teeth may be missing and anodontia becomes evident. Unless some or all of the primary dentition is absent, it is unusual for radiographs to be taken at this age and the diagnosis of hypodontia is often a chance finding.

\section{6-12 years}

Most referrals occur at this age, as late eruption is noticed by the practitioner or commented upon by the patient or parent. It is essential to determine the reasons for failure of tooth eruption, particularly if there is a disturbance of the sequence of tooth eruption. Part 1 of this series shows the normal eruption sequence and dates. Delayed eruption is common in hypodontia patients and should arouse suspicion. At this stage a panoramic radiograph will show the presence or absence of teeth.

In order to provide optimal treatment, early referral is essential.

\section{$12+$ years}

This group includes those older patients who have for some reason slipped through routine examinations that should have detected the condition. Some patients, including adults, may have declined treatment at an earlier age, but have become dissatisfied with their dental appearance and now request treatment. The decision to refer for advice is based on the practitioner's experience and the accessibility of secondary care. Ideally, all patients with congenitally absent teeth (with the exception of third molars) should be referred for advice. In practice, 


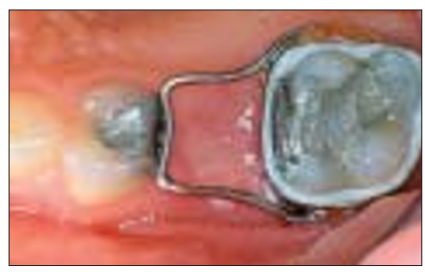

Fig. $1 \mathrm{~A}$ bonded/fixed retainer with a wire loop in situ to prevent mesial drift of the first molar following premature loss of the deciduous second molar

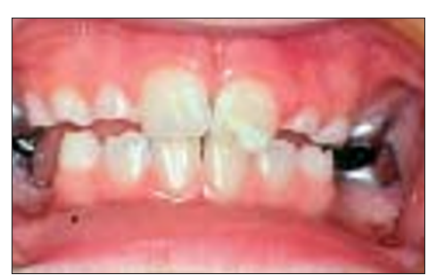

Fig. 2 The first molars have been restored with stainless steel crowns to maintain the posterior occlusion

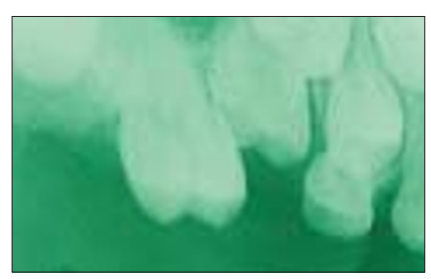

Fig. 3 Infra-occlusion of a primary tooth at the stage where extraction should have occurred previously. The tooth's removal will now require extensive bone loss
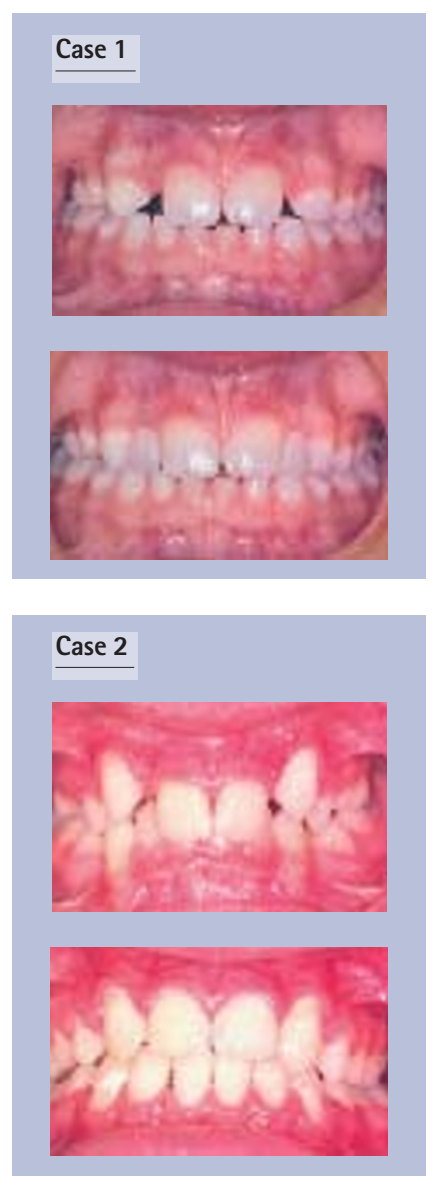

this may not always be possible and absence of a single tooth in a quadrant can often be treated in a straight-forward way. However, the greater the extent of hypodontia, the greater is the need for specialist referral and interdisciplinary care.

\section{MAINTENANCE OF EXISTING DENTITION}

In hypodontia it is usually desirable that patients retain their existing teeth, both primary and permanent. The general dental practitioner's role in caries prevention and maintenance of primary teeth is important. Teeth are necessary for the maintenance and normal development and maintenance of alveolar bone, which in turn is essential for implant placement. Primary teeth act as ideal space maintainers that prevent the undesirable movement of adjacent teeth, which may cause later difficulties in placement of fixed prostheses. The use of stainless steel crowns and appropriate space maintenance may be indicated (Figs 1 and 2).

The dental practitioner is in the best position to monitor the progress of infra-occlusion ('submergence') of primary teeth. Extraction of an infra-occluding primary tooth is indicated when the tooth is below the interproximal contact points of adjacent teeth and before it reaches the point where extraction of the ankylosed tooth would result in excessive alveolar bone destruction (Fig. 3).

\section{Treatment}

Practitioners may be involved in treatment provision, ideally as part of the interdisciplinary approach but sometimes on their own. In all cases of hypodontia there are essentially four treatment options:

1. Accept the situation with no intervention, and provide maintenance.

2. Close the space orthodontically or by elective extraction of primary teeth.

3. Maintain the space and restore it by either dentures, bridges, tooth transplantation or implants.

4. Redistribute the space in order to facilitate prosthetic replacement of the absent teeth.

One of the difficulties in treatment planning for hypodontia patients is deciding what is simple and what is complex, a decision that often requires expert knowledge and skill. It is therefore important for general practitioners to be aware of limitations of their experience and to refer for advice. The number of teeth missing is an extremely poor indicator of when to refer and of treatment complexity. As shown in the previous part of this series, those cases that superficially appears to be simple, can be very challenging and caution is stressed when a treatment plan is devised without reference to other dental disciplines.

We would suggest that most practitioners would wish to limit their management of hypodontia cases, without referral for opinion, to patients with adequate space available for replacement of the absent tooth. In addition, there were no occlusal interferences on excursion or there were no malocclusion present.

The following cases will illustrate how the general dental practitioner can interact with an interdisciplinary team in the management of hypodontia.

\section{SIMPLE HYPODONTIA}

These are cases that an experienced general dental practitioner may be able to plan and treat independently.

\section{Case 1}

UR2 (12) and UL2 (22) are absent but with adequate space. There is adequate space to replace the absent teeth and the occlusion is class I with no crowding, Treatment is simple - resinretained bridges to replace the missing UR2 (12) and UL2 (22).

\section{Case 2}

A similar case of absent upper lateral incisor teeth. However, there is a degree of crowding and a decision will have to be made whether to close or maintain the space. In this case space analysis is required (as with many patients seen on a hypodontia clinic) and a diagnostic wax up is invaluable. The decision was made to close the spaces with fixed orthodontic appliances, aligning the canines to replace the lateral incisors.

When making this decision, the colour, size and shape of the canine are important. To make a good replacement for a lateral incisor, the canine needs to be small mesio-distally, not bulbous in shape and the colour should be similar to the central incisor. To mimic the canines, the premolars are over-rotated carefully to hide the palatal cusp. In this case, the canine shape was not ideal, with long clinical crown, pointed cusp and bulbous shape. Further improvement to shape and colour is often possible through a combination of enamel reshaping and adhesive composite restorations. $^{2}$

\section{Case 3}

This case illustrates a poor aesthetic outcome resulting from the unplanned loss of primary teeth and emphasises the need for early recognition and referral.

A potentially simple case involving the absence of the UL2 (22) has converted into one requiring complex tooth movements. Loss of URC (53) has allowed UR4 (14) to drift mesially and rotate, reducing the space available for the palatally displaced UR3 (13). The LR3 has over erupted, further reducing the available space. On the left, the UL3 (23) has erupted into the UL2 (22) space and there is thus residual space between the canine and premolar. Treatment will require appliance therapy to align the ectopic canine and redistribute the space prior to prosthetic replacements.

In brief, the general practitioner's role in simple cases is: 
- Referral if appropriate

- Provisional diagnosis and maintenance

- Treatment planning

- Treatment in some cases

- Maintenance of restorations/preventive dental care

\section{MODERATE HYPODONTIA}

These are patients with one or more absent teeth in one quadrant, or absent teeth in all quadrants and it is now that the difficulty in treatment planning increases.

\section{Case 4}

Shows a patient with absent, UR5 (15), LL5 (35) LR5 (45)and LL1 (31).

The primary molar teeth are retained and there is no crowding. Treatment was aimed at maintaining the primary teeth, with a view to their later replacement with a mix of implants and resin-bonded bridges.

\section{Case 5}

Case 5 shows a patient with absent teeth in one arch similar to Case 4 yet on closer examination, there are problems with space requirements; the absent teeth result in a large span for bridgework. In addition, there is sufficient crowding in the opposite arch to warrant extraction. In view of the success rate of transplants, the lower extracted premolars were transplanted into the available space in the upper arch and orthodontic treatment used to align the lower arch. Following endodontic treatment and bridge work, treatment will be complete.

The role of the general dental practitioner in moderate cases is:

\section{- Referral}

- Treatment, often restorative eg placement of bridges and/or reshaping of teeth

- Maintenance.

\section{SEVERE HYPODONTIA}

These cases present the greatest challenge to the interdisciplinary team. Achieving an ideal occlusion may not be possible and often a compromise has to be reached by negotiation between the team members representing the various dental disciplines. Some cases are relatively easy to plan, but technically difficult to deliver and vice versa.

\section{Case 6}

Case 6 shows a case of severe hypodontia, with teeth missing in all quadrants. However, the treatment planning is relatively simple.

The spaces between permanent teeth are almost optimal and adhesive bridgework is the method of choice to replace the absent teeth, with composite build ups of the incisors after closure of the median diastema.

\section{Case 7}

Case 7 illustrates a difficult planning case. There are a several treatment planning decisions to be made:
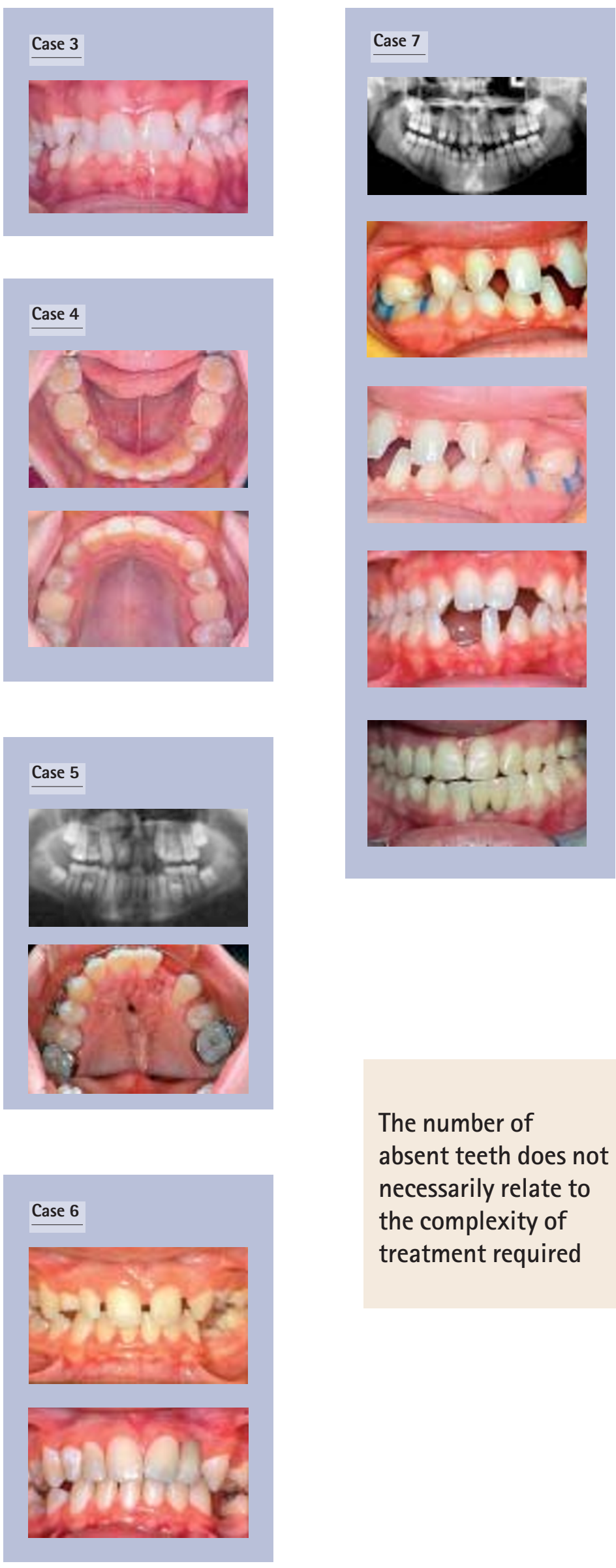

The number of absent teeth does not necessarily relate to the complexity of treatment required 


\section{PRACTICE}

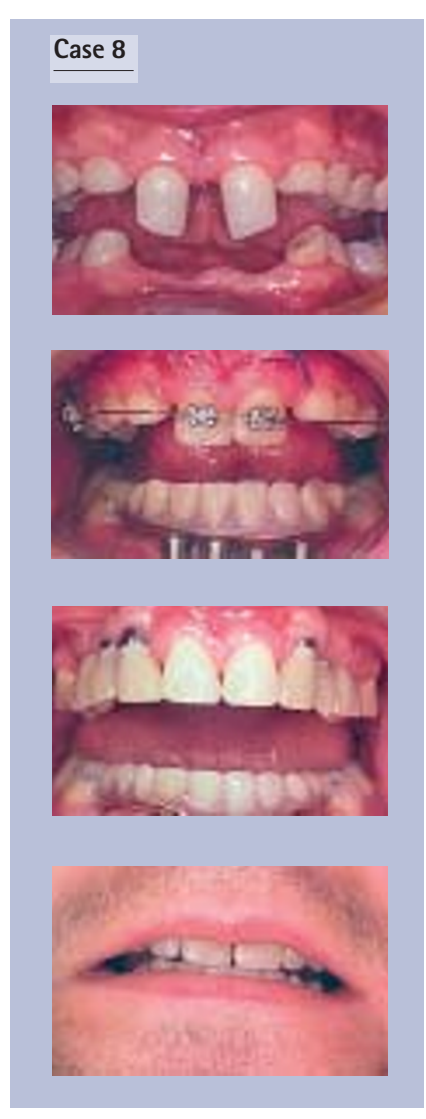

- Open the spaces to facilitate bridges?

- Close the spaces orthodontically?

- If the spaces are opened, are bridges preferable to implants?

- Is permanent post-orthodontic retention needed?

- Is there sufficient bone for implants?

In these cases a series of diagnostic wax-ups will help the treatment planning process. As can be seen, the option chosen was to optimize the spaces orthodontically for prosthetic replacements.

\section{Case 8}

Case 8 shows what appears to be a simple case that taxes the skills of the whole team. The patient has a large number of absent teeth in all quadrants with only UL6 (26), UL1 (21), UR1 (11) UR6 (16), LL6 (36), LR6 (46) present. There is a significant overbite of the anterior teeth, and there are retained primary teeth.

The treatment requires, at the outset, intensive oral hygiene efforts, orthodontic movement to optimize mesio-distal and interocclusal space, extraction of the primary teeth, sinus lift bone augmentation, implant bridge-work and occlusal onlays to complete treatment.

When considering implants it is extremely important to ascertain the positions of the roots prior to removal of orthodontic appliances. This is because the use of orthodontic pre-adjusted appliances positions the crowns of teeth to the ideal, rather than paralleling the roots. This latter consideration is important for the abutment teeth to ensure that they do not intrude upon space required for an implant. This patient was treated a number of years ago using angulated abutments for screw-retained bridges with obvious compromised results.

In severe hypodontia the general dental practitioner can work with the team in the essential role of:

- Undertaking routine dental care to maintain the existing dentition

- Assist with treatment as appropriate

- Maintenance following completion of treatment.

This series of papers has shown how an interdisciplinary team can work to provide the best treatment for the hypodontia patient, in many cases in a complementary way with the patient's general dental practitioner.

1 Nunn J H, Carter N E, Gillgrass T J, Hobson R S, Jepson N J, Meechan J G, Nohl FS. The interdisciplinary management of hypodontia, Part 1: background and role of paediatric dentistry. Br Dent J2003; 194: 245-256.

2 N. J. Jepson, F. S. Nohl, N. E. Carter, T. J. Gillgrass, J. G. Meechan, R. S. Hobson, J. H. Nunn. The interdisciplinary management of hypodontia, Part 2: restorative dentistry. BrDent J 2003; 194: 299-304.

\section{ONLINE SUBMISSION to the British Dental Journal}

From July 2002, the British Dental Journal at www.bdj.co.uk is pleased to be able to offer its authors the option to submit their manuscripts online.

- Authors from anywhere in the world will be able to quickly and easily enter their contact details into an online form, and attach their manuscript files, either as separate text and graphics, or as an integrated file.

- Author files will be automatically converted into a PDF (Portable Document Format) file, which can be approved by the author on screen prior to submission. Submissions will be promptly acknowledged by e-mail. Editors and referees will then view the PDF on the website - cutting out the time that manuscripts traditionally spend in the postal system.

- Authors who, for whatever reason, are unable to submit online can also benefit from the timeliness of electronic peer review. Authors are encouraged to submit their manuscripts on disk, or if necessary hard copy submissions can be converted into PDF files using highquality scanners.

- Making use of online submission and electronic peer review will enable us to speed up the review process, providing a better and more efficient service to authors.

For further information about submitting your paper electronically please e-mail:

$$
\text { k.maynard@bda-dentistry.org.uk }
$$

